\title{
Erratum to: Impact of hybrid procedure on $P$ wave duration for atrial fibrillation ablation
}

\author{
Narendra Kumar • Pietro Bonizzi • Laurent Pison • \\ Kevin Phan • Theo Lankveld • Bart Maesen • Mark La Meir • \\ Sandro Gelsomino • Jos Maessen • Harry Crijns
}

Published online: 18 February 2015

(C) Springer Science+Business Media New York 2015

Erratum to: J Interv Card Electrophysiol (2015)

DOI 10.1007/s10840-014-9969-9

The original version of this article unfortunately contained a mistake, Bart Maesen was spelled incorrectly.

The online version of the original article can be found at http://dx.doi.org/ 10.1007/s10840-014-9969-9.

N. Kumar $(\bowtie) \cdot$ L. Pison $\cdot$ H. Crijns

Department of Cardiology, Maastricht University Medical Centre and Cardiovascular Research Institute Maastricht (CARIM), P.

Debyelaan 25, PO Box 5800, 6202AZ Maastricht, The Netherlands

e-mail: drnarendra007kr@gmail.com

B. Maesen · M. La Meir · S. Gelsomino · J. Maessen

Department of Cardiac Surgery, Maastricht University Medical

Centre and Cardiovascular Research Institute Maastricht (CARIM),

Maastricht, The Netherlands

T. Lankveld

Department of Physiology, Maastricht University Medical Centre and Cardiovascular Research Institute Maastricht (CARIM),

Maastricht, The Netherlands

P. Bonizzi

Department of Knowledge Engineering, Maastricht University, Maastricht, The Netherlands

K. Phan

Macquarie University and Westmead Hospital, Sydney Medical

School, University of Sydney, Sydney, Australia

S. Gelsomino

Department of Heart and Vessels, Careggi Hospital, Via Delle Oblate

1, 50134 Florence, Italy 